\title{
Formation of Resin-Rich Zones in Composites Processing
}

\author{
Chensong Dong ${ }^{1, a}$ and Tze Chiun Tsai ${ }^{1, b}$ \\ ${ }^{1}$ Department of Mechanical Engineering, Curtin University of Technology, GPO Box U1987, Perth, \\ WA 6845, Australia \\ ac.dong@curtin.edu.au, ${ }^{b} \underline{\text { tzechiun.tsai@student.curtin.edu.au }}$
}

Keywords: Composites; deformation, resin-rich

\begin{abstract}
Resin-rich zones are a common phenomenon in liquid composite molding processes. These resin-rich zones cause unwanted residual stress and deformation, and part-to-part variation, and thus they need to be studied in the design of composite structures. An experimental study on the formation of resin-rich zones in angled composite parts is presented in this paper. Two openchannel mold sets were designed and fabricated. Fiber preforms were loaded into these molds and the gaps formed were visually inspected by a microscope. The influences of corner radius, fiber volume fraction, enclosed angle, and stacking sequence were investigated, and significant factors affecting gap thickness were identified by Design of Experiments (DOE). It can be concluded from the experimental results that:

1) Fiber volume fraction is the most significant factor affecting gap thickness. Gap thickness is inversely proportional to fiber volume fraction;

2) Gap thickness is inversely proportional to radius;

3) The gap thickness of unidirectional preforms is larger than that of the cross-ply preforms.
\end{abstract}

\section{Introduction}

Fiber-reinforced composites are widely used in many structural components in aircraft, automotive, marine, and other industries [1] for their advantages of low density, high strength, high stiffness to weight ratio, excellent durability, and design flexibility. Despite all these advantages, composite materials have not been as widely used as expected because of the complexity and cost of the manufacturing process. One of the main problems is poor dimensional control.

The typical process-induced dimensional variation for angled composite parts is called spring-in, which is the reduced enclosed angle. The spring-in of composites was either studied by elastic [2] or viscoelastic [3] models.

Resin-rich zones are a common phenomenon in liquid composite molding processes. These resinrich zones cause unwanted residual stress and deformation, and part-to-part variation, and thus they need to be studied in the design of composite structures. In resin transfer molding processes, dry fiber preform is compressed during mold closure. For an angled part, this compression pulls the fibers at the corner radius tight and a gap is formed between the fiber preform and the mold surface. After resin infusion, a resin-rich zone is formed. Holmberg et al. [4] studied the manufacturing and performance of RTM U-beams. As the mould closes, the reinforcement tends to pull tight around corners and leaves a resin-rich area.

When resin-rich zones exist, spring-in cannot be directly calculated by these models, and numerical methods such as finite element analysis are needed. Accurate modeling of spring-in requires that the sizes and locations of resin-rich zones can be predicted. The literature survey shows that there is no study on resin-rich zones and their influence on spring-in.

To fill this technical gap, this paper presents an experimental study on the formation of resin-rich zones in angled composite parts. Two open-channel mold sets were designed and fabricated. Fiber preforms were loaded into these molds and the gaps formed were visually inspected by a microscope. The influences of corner radius, fiber volume fraction, enclosed angle, and stacking sequence were investigated, and the significant factors affecting gap thicknesss were identified by Design of Experiments (DOE). 


\section{Experiments}

Previous research [5] shows that spring-in decreases with resin-rich layer thickness. In this study, gaps between fiber preform and internal mold surface were investigated experimentally to improve our understanding of the mechanism of formation of resin-rich zones. Open channel molds were designed and made for the convenience of visual observation. Dry fiber mats were loaded into the open channel mold cavity and the gaps formed between the fiber preform and internal mold surface were examined under a microscope.

Two categories of factors affecting resin-rich zones were identified, i.e. geometric and materials parameters. A $2^{4}$ factorial design was chosen all possible combinations of the levels of the factors are investigated [6]. The levels of variables are shown in Table 1.

Table 1: Levels of variables

\begin{tabular}{lcc}
\hline Name & Low & High \\
\hline Radius, $r(\mathrm{~mm})$ & 5 & 10 \\
Fibre volume fraction, $V_{f}$ & 0.39 & 0.58 \\
Enclosed angle, $\phi\left(^{\circ}\right)$ & 90 & 135 \\
Stacking sequence & $0^{\circ}$ unidirectional & cross-ply \\
\hline
\end{tabular}

A number of levels were chosen for each factor. As shown in Fig. 1, the geometric parameters include enclosed angle, corner radius, and thickness. Since spring-in is a function of radius/thickness ratio, in this study, a fixed laminate thickness $4 \mathrm{~mm}$ was chosen and only corner radius was varied. Thus, two corner radius levels 5 and $10 \mathrm{~mm}$ were chosen. The enclosed angle levels were chosen to be $90^{\circ}$ and $135^{\circ}$.

The experiment set up is shown in Fig. 2. The fiber preform of a specific stacking sequence was prepared by cutting and stacking a number of E-glass fiber mats. The prepared preform was then load into the mold cavity. After the mold was closed and clamped, the fiber preform in the mold cavity was examined by a microscope.

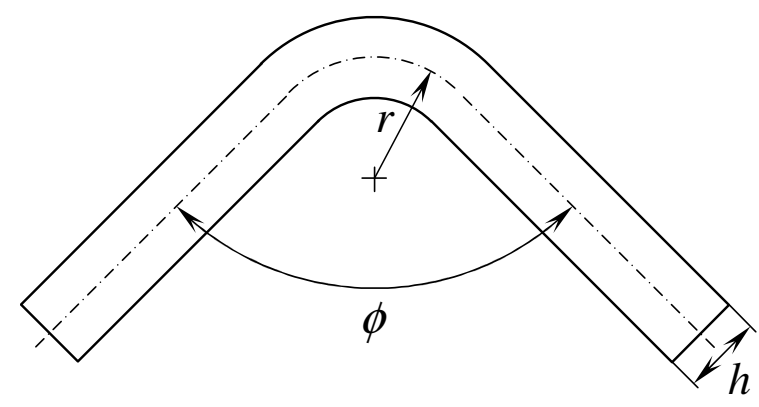

Fig. 1: An angled composite part

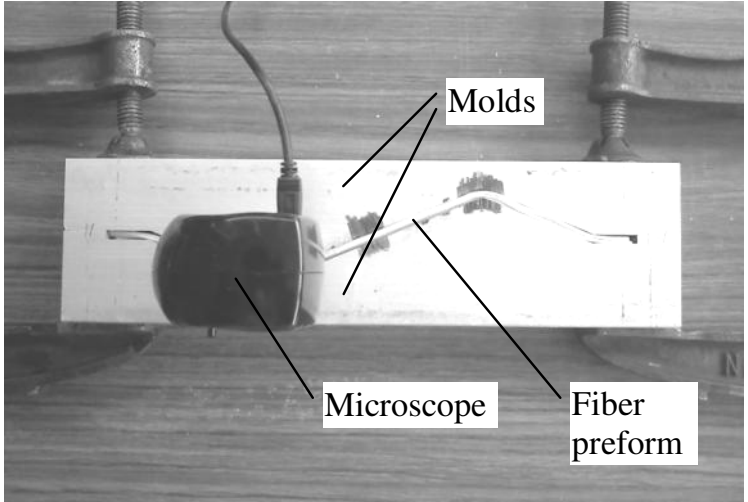

Fig. 2: Experiment setup

\section{Results and Discussion}

Fig. 3 shows two examples of gaps formed at the corner radii. The data from experiments were analyzed by Design-Expert ${ }^{\circledR}$ software (Stat-Ease, Inc. Minneapolis, MN). Fig. 4 shows the percent contributions of effects, from which it can be seen that fiber volume fraction is the most significant factor affecting maximum gap thickness. Maximum gap thickness is also strongly dependent on stacking sequence and radius. It can be seen that maximum gap thickness is inversely proportional to fiber volume fraction and radius. The maximum gap thickness of unidirectional preforms is larger than that of cross-ply preforms. 


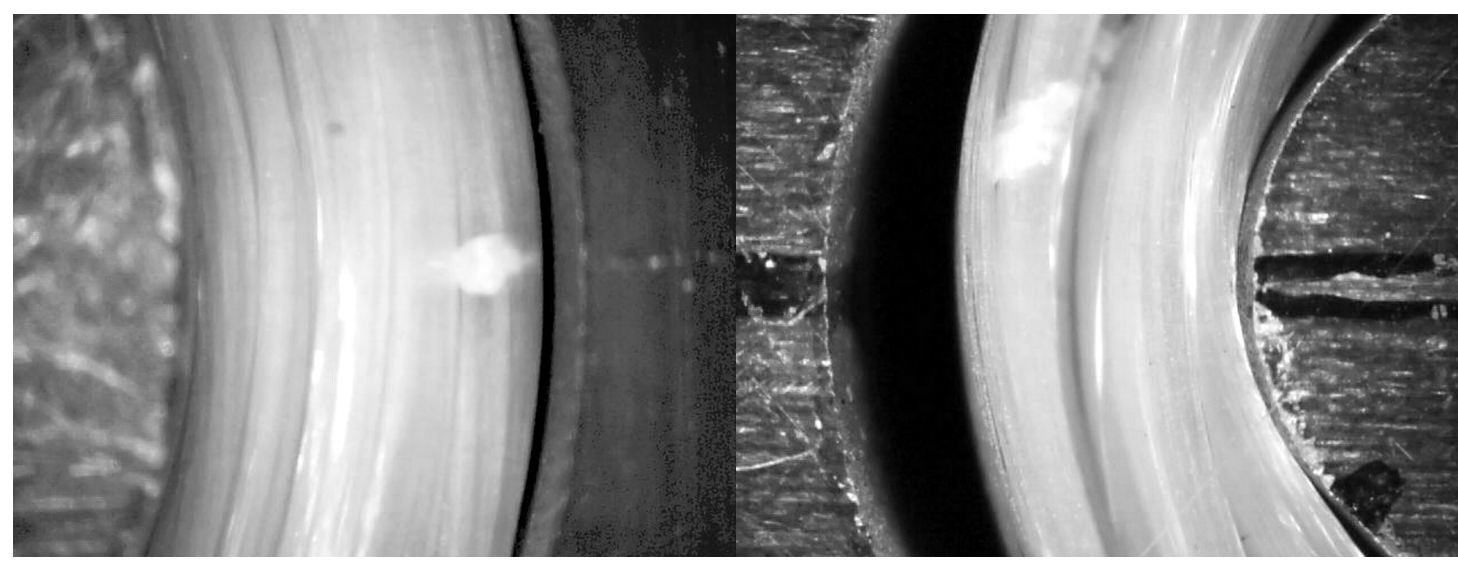

Fig. 3: Gaps at corner radius

Left: $0^{\circ}$ unidirectional; $\phi=135^{\circ} ; V_{f}=0.58 ; r=10 \mathrm{~mm}$; right: $0^{\circ}$ unidirectional; $\phi=90^{\circ} ; V_{f}=0.39 ; r=5 \mathrm{~mm}$

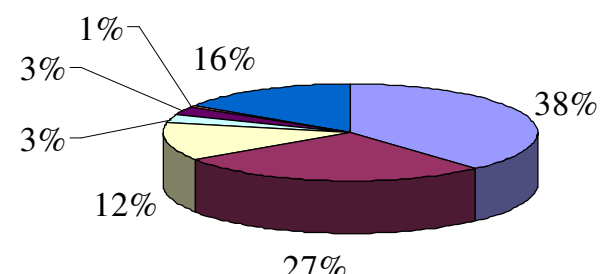

$\square$ Fiber volume fraction

$\square$ Stacking sequence

$\square$ Radius

$\square$ Fiber volume fraction-stacking sequence interaction

$\square$ Radius-enclosed angle interaction

$\square$ Enclosed angle

$\square$ Others

Fig. 4: Percent contributions of effects

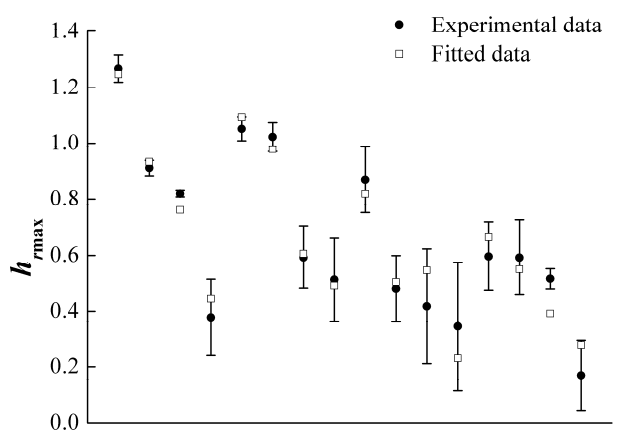

Fig. 5: Fitted data vs. experimental data

Two regression models were fit to the data as:

For $0^{\circ}$ unidirectional preforms:

$$
h_{r \max }=3.27414-0.14323 r-2.56786 V_{f}-7.88015 \times 10^{-3} \phi+8.90504 \times 10^{-4} r \phi
$$

For cross-ply preforms:

$$
h_{r \max }=2.40374-0.14323 r-1.43636 V_{f}-7.88015 \times 10^{-3} \phi+8.90504 \times 10^{-4} r \phi
$$

The fitted and experimental data are shown in Fig. 5, from which it can be seen that the models can well interpret the experimental data.

Fig. 6 shows the mechanism of the formation of gaps at corner radius. When mold is closed, pressure $P$ is induced and it causes friction force $f$. The resultant pressure $F$ due to the friction force $f$ compresses the fiber preform at the corner radius and a gap is formed.

The size of gap is dependent on the friction force and the compressibility of fiber preform. When fiber volume fraction is higher, there are more fibers and fiber preform is more compacted. Thus, the compressibility of fiber preform is lower, and gap decreases. When radius decreases, the pressure increases because of the decreased area, thus gap increases. Because all the fibers of $0^{\circ}$ unidirectional fiber preforms are oriented in the same direction, their compressibility is higher than that of cross-ply fiber preforms. Thus, the gap thickness of $0^{\circ}$ unidirectional fiber preforms is larger than that of cross-ply fiber preforms. 


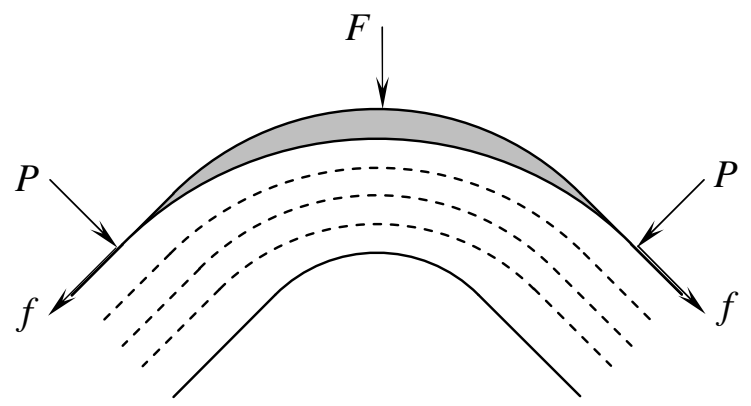

Fig. 6: Gap formation at corner radius

\section{Conclusions}

This paper presents an experimental study on the formation of resin-rich zones in angled composite parts. Two open-channel mold sets were designed and fabricated. Fiber preforms were loaded into these molds and the gaps formed were visually inspected by a microscope. It is found from the experiments that gaps are formed at corner radii; and beside corner radii when the fiber volume fraction is low. The influences of corner radius, fiber volume fraction, enclosed angle, and stacking sequence have been investigated, and the significant factors affecting gap thickness were identified by Design of Experiments (DOE). It can be concluded from the experimental results that:

1) Fiber volume fraction is the most significant factor affecting the gap thickness at corner radius. Gap thickness is inversely proportional to fiber volume fraction;

2) Gap thickness is inversely proportional to radius;

3) The gap thickness of unidirectional preforms is larger than that of the cross-ply preforms.

\section{Acknowledgment}

Chensong Dong thanks the support from the Curtin Research Fellowship.

\section{References}

[1] P. K. Mallick: Fiber-Reinforced Composites: Materials, Manufacturing, and Design (Marcel Dekker, New York 1993).

[2] D. W. Radford, R. J. Diefendorf: Journal of Reinforced Plastics and Composites Vol. 12 (1993), p. $58-75$.

[3] J. M. Svanberg, J. A. Holmberg: Composites Part A Vol. 35 (2004), p. 711-721.

[4] J. A. Holmberg, L. A. Berglund: Composites Part A Vol. 28 (1997) p. 513-521.

[5] C. Dong, in: 11th Japan International SAMPE Symposium \& Exhibition, Tokyo, Japan (2009).

[6] D. C. Montgomery: Design and Analysis of Experiments, 5th Edition (John Wiley \& Sons, Inc., New York 2000). 